\title{
Un pionnier de la médecine sociale et du travail social en hôpital
}

\section{Jean Martin}

Dr méd., membre de la rédaction

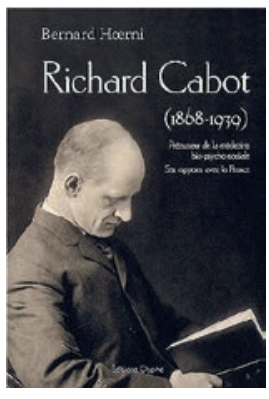

Bernard Hœrni

Richard Cabot (1868-1939)

Précurseur de la médecine

bio-psycho-sociale

Paris: Editions Glyphe, 2019, 164 p.

Richard Cabot est un médecin né à Boston, d'une famille d'intellectuels, et œuvra en laboratoire (hématologie) avant de devenir professeur de médecine à Harvard - puis d'éthique sociale à la fin de sa carrière. Bernard Hœrni, professeur émérite de Bordeaux, s'est beaucoup intéressé à Cabot, considérant que son œuvre était injustement méconnue.

\section{Une vision de santé communautaire}

$\mathrm{Au}$ Massachussetts General Hospital, Cabot transforme la manière dont la policlinique (outpatient department) était organisée, sur la base d'une vision globale selon laquelle les conditions économiques, sociales, familiales et psychologiques sous-tendent beaucoup les affections dont souffrent les patients: «Il faut reconstituer l'historique des désordres, leurs circonstances. Malgré ce qu'expose souvent le patient, les troubles actuels sont rarement accidentels*.» Il évoque des situations illustratives: la tuberculose et son traitement (cette maladie est l'exemple de ce que William Osler appelait un "problème social avec des aspects médicaux», multifactoriel); Comment les neurasthéniques peuvent-ils retrouver équilibre et bonheur? Comment aider une femme ayant un enfant sans mari? Dans ces cas tabous à l'époque, il est toutefois réservé quant à une prise en charge en institution.

Ce qui me rappelle (J.M. cette formule discutée avec les étudiants, «L'accident n'est pas accidentel»-il survient par la conjonction de divers facteurs de risque.

jean.martin[at]saez.ch

\section{Le travail social à l'hôpital}

Cabot met en place un système où des assistantes sociales œuvrent en complémentarité des médecins et montre que cela améliore les résultats du traitement, en apportant une perspective critique. Il crée en 1905 les premières positions d'assistantes sociales professionnelles, collaborant durant quarante ans avec Ida Maud Cannon, sœur du physiologiste Walter Cannon.
Et comme l'hôpital refusait de payer ces dernières, il le fait de sa poche. En 1917, il rejoint le corps expéditionnaire américain en France, où il travaillera dix-huit mois; son livre Essais de médecine sociale y paraît en 1919. 11 soutient également la création de l'école d'infirmières laïques de Bordeaux.

Bernard Hœrni cite un contemporain de Cabot: «Partout où fonctionne l'organisation du Dr Cabot, l'hôpital subit une transformation. Il y a une atmosphère de confiance.»

\section{Pratique et éthique médicales}

Les constats faits à l'époque par Cabot restent d'actualité aujourd'hui: «Il y a des siècles, écrit-il, que les médecins caressent l'idéal de sonder aussi loin que possible les causes de la maladie. Mais leurs formation et caractère professionnels les incitaient à se concentrer sur les manifestations physiques et à négliger l'intelligence et la volonté du patient, ainsi que ses réactions à diverses dimensions de son milieu [...] A l'hôpital on ne voit le malade qu'arraché à son entourage naturel.» Très en avance sur son temps, à une époque où l'information aux malades reste dominée par le «mensonge médical» voire dit «thérapeutique» (!), Cabot défend le droit des patients à une information complète et exacte: il faut leur faire confiance, ils sont capables d'entendre des nouvelles désagréables. «Je n’ai jamais vu un malade s'aggraver en apprenant la nature de sa maladie.»

Pionnier de la formation professionnelle continue, il s'implique pour convaincre qu'on peut s'instruire à partir de ses erreurs et met sur pied les conférences anatomo-cliniques.

\section{Préoccupations écologiques}

Cabot affirme également des conceptions écologiques avant l'heure, relevant que l'homme emprunte beaucoup à la nature mais, comme il en fait partie, il doit respecter un équilibre entre ce qu'il reçoit et ce qu'il donne. Bien écrite, vivante, cette biographie suit en détail l'itinéraire d'un pionnier de la prise en compte des conditions sociales en médecine. Elle fourmille d'informations sociales et historiques sur la vie de Richard Cabot, son milieu, les Etats-Unis à la fin du XIX siècle et au début du XXe. Une contribution bienvenue. 